\title{
Projected change in extreme rainfall events in China by the end of the 21st century using CMIP5 models
}

\author{
CHEN HuoPo \\ Nansen-Zhu International Research Centre (NZC), Institute of Atmospheric Physics, Chinese Academy of Sciences, Beijing 100029, China
}

Received July 1, 2012; accepted September 12, 2012; published online January 11, 2013

Projection of future climate changes and their regional impact is critical for long-term planning at the national and regional levels aimed at adaptation and mitigation. This study assesses the future changes in precipitation in China and the associated atmospheric circulation patterns using the Couple Model Intercomparison Project 5 Phase (CMIP5) simulations under the RCP4.5 and RCP8.5 scenarios. The results consistently indicate that the annual precipitation in China is projected to significantly increase at the end of the 21 st century compared to the present-day levels. The number of days and the intensity of medium rain, large rain and heavy rain are obviously increased, while the number of trace rain days is projected to decrease over the entire area of China. Further analysis indicates that the significant increase of annual precipitation in Northwest China is primarily due to the increase of light rain and the increases in North and Northeast China are primarily due to the increase of medium rain. In the region of southern China, the increases of large rain and heavy rain play an important role in the increase of annual precipitation, while light rain events play a negative role. Analysis of the changes in atmospheric circulation indicates that the East Asian summer monsoon circulation is projected to be considerably stronger, and the local atmospheric stratification is projected to be more unstable, all of which provide a background benefit for the increase of precipitation and extreme rainfall events in China under global warming scenarios.

CMIP5, precipitation, extreme rainfall, atmospheric circulation, projection

Citation: Chen H P. Projected change in extreme rainfall events in China by the end of the 21st century using CMIP5 models. Chin Sci Bull, 2013, 58: 1462-1472, doi: $10.1007 / \mathrm{s} 11434-012-5612-2$

The significant warming that has been observed globally over the past century has a high probability of being due to the increase in anthropogenic factors concentrating greenhouse gases. This significant warming has produced a significant impact on human society, ecosystems, and the environment, and this warming is capable of causing thousands of deaths and heavy economic losses. By the end of the 21st century, as reported in the Fourth Assessment Report of the Intergovernmental Panel on Climate Change (IPCC AR4), human society will become much more vulnerable as the consequences of continuous global warming, with global temperature increasing by $1.1-6.4^{\circ} \mathrm{C}$ relative to 1980-1999 [1]. Due to these future negative impacts, governments and the public alike have begun to pay increased

email: chenhuopo@mail.iap.ac.cn attention to climate change under the global warming scenario.

Modern global climate models that are capable of realistically simulating many aspects of the climate are the primary tools for determining the attribution of recent warming and for developing projections of future climate change. Researchers in China have taken a great deal of effort to study future climate change based on such model simulations, and some valuable and consistent results have recently been obtained [2-9]. For example, based on the multi-model ensemble mean (MME) of 16 CMIP3 model simulations, Jiang and $\mathrm{Fu}[10]$ showed that the annual mean precipitation is expected to increase on average by $3.4 \%-4.4 \%$ over China with a $2^{\circ} \mathrm{C}$ increase in global temperature. Buhe [11] and Luo et al. [12] also indicated that the summer precipitation in China is projected to see a significant increase 
as a result of global warming. This change is closely associated with the intensified East Asian summer monsoon circulation $[6,13]$. Compared with the climatology, the extreme rainfall events are much more sensitive to the global climate change. Jiang et al. [14] indicated that the events related to precipitation will become much more extreme, and the magnitude of this change is scaled to various emission scenarios. Similar results can be found in Chen et al. [15], who also documented that by the end of the 21st century, the region of eastern China will experience more intense and more frequent heavy rainfall events. They also indicated that the increased probability of heavy rainfall events in this region is closely connected with increased water vapor transportation from the Tropical Ocean and the increasing instability of the atmosphere stratification under the global warming scenario. In addition, the frequency of the intense snowfall events over southern China is reported to decrease, while over northern China the intense snowfall events initially increase and then decrease [16]. These changes exhibit significant agreement with the simulations by regional climate models (RCM), but with more local small-scale information provided in the high-resolution RCMs [17-21]. These results have been well reviewed by Ding et al. [22] and Wang et al. [23].

These previous studies are almost basing on CMIP3 simulations. However, recently, the simulation results from the latest generation couple models have been gradually released and are archived at the website of the Earth System Grid gateway that is hosted by the Program for Climate Model Diagnosis and Intercomparison. This dataset is called the "CMIP5 multi-model dataset" (referred to as CMIP5 in the following), which includes the climate model outputs for IPCC AR5. Compared with the earlier phases, the experimental design and the emission scenarios in CMIP5 seem to be much more reasonable. The primary analysis on climate change using these model outputs has been previously begun $[24,25]$. However, changes in precipitation and its related extremes in China have yet to be investigated using the CMIP5 models. Thus, the purpose of this study is to investigate the future changes in the number of days and the intensity of rainfall events in different intensities, as well as in its corresponding contribution to the increased annual precipitation. The changes in the associated atmospheric circulation patterns are further discussed.

\section{Data and method}

Compared with the emission scenarios in CMIP3, the representative concentration pathways (RCP) related to the radiative forcing are identified as new climate scenarios in CMIP5, and they include RCP2.6, RCP4.5, RCP6.0, and RCP8.5. More information about these new scenarios, as well as the experimental design, can be found in reference
[26]. In this study, we mainly focus on the changes in extreme rainfall events in China at the end of the 21 st century in the RCP4.5 (medium radiative forcing scenario) and RCP8.5 (high radiative forcing scenario) scenarios. Here, RCP4.5 corresponds to the case of radiative forcing after 2100 of approximately $4.5 \mathrm{~W} / \mathrm{m}^{2}$, which is equivalent to approximately $650 \mathrm{ppm} \mathrm{CO}_{2}$, which is somewhat larger than that in the B1 scenario (approximately $550 \mathrm{ppm}$ ) in IPCC AR4. Similarly, RCP8.5 is defined as the case where the radiation is assumed to exceed $8.5 \mathrm{~W} / \mathrm{m}^{2}$, which means the equivalent $\mathrm{CO}_{2}$ exceeds $1370 \mathrm{ppm}$, and it is considerably greater than that in the A2 scenario (860 ppm) [27]. Thus, the daily precipitation outputs from 16 CMIP5 models for the period of 2006-2099 in the RCP4.5 and RCP8.5 scenarios are used here. A brief summary of these models is presented in Table 1. Meanwhile, the historical simulations from 1986-2005 and the site-observed daily precipitation data from 730 stations, which are released by the Chinese National Climate Center, are also used here to evaluate the reproducibility of these models. In addition, to discuss the favorable background for the changes of extreme rainfall, several other variables are analyzed, such as the monthly horizontal winds, specific humidity, relative humidity, atmosphere temperature, 2-m air temperature, precipitable water content, and sea level pressure. Note that the FGOALS-g2 model has not provided the variable of precipitable water content in its website and is thus not included in this discussion of the future change in atmospheric water content. We selected the target period of 1986-2005 as the present-day climate and the period of 2080-2099 as the future. For convenient analysis, all the simulations are bilinearly interpolated into a common grid of $2^{\circ}$ in latitude and $2^{\circ}$ in longitude in this study.

The multi-model ensemble (MME) approach is applied in this study in an effort to reduce the uncertainty from the internal variability and inter-model difference. We only take a simple multiple model mean and the model weight is not considered. This approach has been widely used in future climate projections [1]. Some studies further document that the MME performance is expected to outperform individual models in the case of present-day climate simulation over East Asia [28,29]. Meanwhile, the model reproducibility of the observed precipitation climatology is evaluated, and the results indicate that all the models have good performance in simulating the spatial patterns (Figure not shown) in the target period. The range of spatial correlation coefficients with observations is $0.34-0.85$ (256 grids in China). Furthermore, the MME result was reported prior to the individual models, and its spatial correlation is up to 0.77 , which further supports the use of the MME method in this study.

To investigate the contribution of the changes in different-intensity rainfall events to the change in annual precipitation, the rainfall events are divided into five categories: trace rain $(0.1-1.0 \mathrm{~mm} / \mathrm{d})$, light rain $(1.0-10 \mathrm{~mm} / \mathrm{d})$, medium 
Table 1 Percentage changes from the period of 1986-2005 to the period of 2080-2099 in China for the average annual precipitation $(P)$, rainy days (Days), intensity $(I)$, and absolute changes in surface air temperature $\left(T_{\text {as }}\right)$ in the RCP4.5 scenario

\begin{tabular}{lllrrrr}
\hline \multicolumn{1}{c}{ Models } & Country & $\begin{array}{c}\text { Atmosphere resolution } \\
\text { (Grids in longitude by latitude) }\end{array}$ & $P(\%)$ & Days (\%) & $I(\%)$ & $T_{\text {as }}\left({ }^{\circ} \mathrm{C}\right)$ \\
\hline BCC-CSM1.1 & China & $128 \times 64$ & 8.2 & -0.5 & 8.7 & 2.1 \\
CanESM2 & Canada & $128 \times 64$ & 16.6 & 5.7 & 12.1 & 3.0 \\
CCSM4 & USA & $288 \times 192$ & 6.6 & -0.7 & 7.7 & 2.1 \\
CSIRO-MK3.6.0 & Canada & $192 \times 96$ & 2.2 & -4.2 & 6.8 & 3.0 \\
FGOALS-g2 & China & $128 \times 60$ & 5.4 & -0.0 & 5.4 & 1.6 \\
FGOALS-s2 & China & $128 \times 108$ & 7.2 & 0.2 & 8.0 & 1.7 \\
HadGEM2-CC & UK & $192 \times 145$ & 15.8 & 1.5 & 15.6 & 3.4 \\
INMCM4 & Russia & $180 \times 120$ & 3.3 & 0.1 & 3.0 & 1.5 \\
IPSL-CM5A-LR & France & $96 \times 96$ & 6.8 & -2.8 & 9.8 & 2.9 \\
IPSL-CM5A-MR & France & $144 \times 143$ & 2.3 & -6.3 & 8.4 & 3.1 \\
MIROC5 & Japan & $256 \times 128$ & 13.5 & 2.4 & 10.6 & 2.8 \\
MIROC-ESM & Japan & $128 \times 64$ & 11.3 & 0.4 & 10.3 & 3.7 \\
MIROC-ESM-CHEM & Japan & $128 \times 64$ & 11.0 & 0.2 & 10.3 & 3.8 \\
MPI-ESM-LR & Germany & $192 \times 96$ & 4.9 & -0.6 & 8.5 & 2.2 \\
MRI-CGCM3 & Japan & $320 \times 160$ & 13.5 & 3.2 & 12.4 & 1.9 \\
NorESM1-M & Norway & $144 \times 96$ & 10.1 & -0.9 & 1.1 & 2.6 \\
Ensemble & & & 8.8 & -0.3 & 9.3 & 2.6 \\
\hline
\end{tabular}

rain $(10-25 \mathrm{~mm} / \mathrm{d})$, large rain $(25-50 \mathrm{~mm} / \mathrm{d})$, and heavy rain $(>50 \mathrm{~mm} / \mathrm{d})$. This categorization method has been widely used in previous studies and in other operational works [30]. The purpose of this study is to investigate the future changes in frequency, intensity, and contribution for each category by the end of the 21 st century, with respect to the present-day climate. The frequency is defined as the number of days that the daily precipitation is involved in the range of each category, and the intensity is the average precipitation for the corresponding category. The contribution is defined as the percent ratio that the precipitation changes for each category, and this figure is then divided by the annual precipitation change.

\section{Future changes in extreme rainfall events}

Figure 1 shows the mean distributions of the daily precipitation frequency (Figure 1(a)) and amount ratio (Figure 1(c)) for the different-intensity categories derived from observations (1986-2005) and the 16-model ensemble simulations for the present (1986-2005, historical) and future (20802099) climates under the RCP4.5 and RCP8.5 scenarios (intensity interval size is $1 \mathrm{~mm} / \mathrm{d}$ ). The percentage changes for the future scenarios are shown in Figure 1(b) and (d). From Figure 1, we can see that MME overestimates the frequency for daily precipitation less than $\sim 15 \mathrm{~mm} / \mathrm{d}$ but underestimates the frequency for precipitation over $\sim 15$ $\mathrm{mm} / \mathrm{d}$. Thus, the amount of intense precipitation (approximately $>15 \mathrm{~mm} / \mathrm{d}$ ) and its contribution to the total precipitation are higher in the observations than in the MME. This feature can be found in each CMIP5 model that was used in this study. Further analysis indicates that the rainfall events in China are projected to be considerably more extreme under global warming scenarios. The number of days of intense precipitation is reported to significantly increase with respect to the present-day, and its contribution to the annual precipitation change is also obviously increased. This magnitude of change is scaled to the emission scenarios, with a greater increase in magnitude of RCP8.5 and a smaller increase in RCP4.5. This change suggests an increased risk of flash floods in China from increased levels of intense precipitation under the RCP emission scenarios.

The previous studies consistently document that the amount of precipitation in China is significantly increased under global warming scenario [2,6]. Similar results can be found in the CMIP5 simulations. Figure 2(a) shows the distribution of the percentage changes in the annual precipitation by the end of the 21 st century under the RCP4.5 scenario with respect to the present-day simulation. It is clear that the annual precipitation is projected to increase in the whole of China, with a larger increase in northern China but with a smaller increase in southern China. Further analysis indicates that a consistent increase of annual precipitation in northern China can be found in over $90 \%$ of the models. Considering the average across China, the likely range of increase in the annual precipitation is projected to be $2.2 \%-$ $16.6 \%$ for the 16 CMIP5 models and $8.8 \%$ in the MME result (Table 1) under the RCP4.5 scenario. This increase in magnitude for RCP8.5 is much larger and the likely range of increase is $2.8 \%-25.8 \%$ for the 16 CMIP5 models and $13.9 \%$ in the MME. Some differences can be found between 

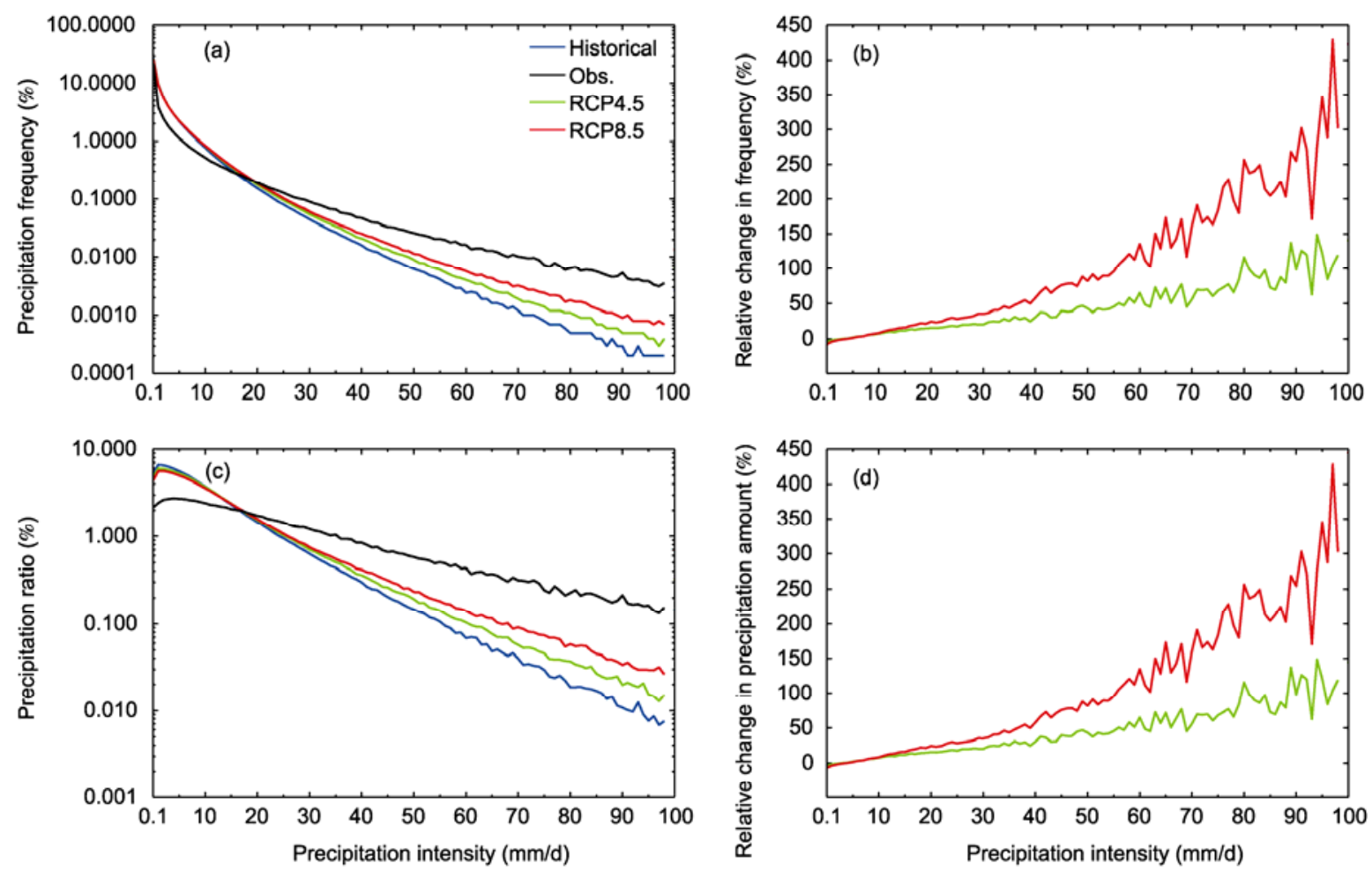

Figure 1 (a) China average distribution of the daily precipitation frequency as a function of the precipitation intensity (interval size is $1 \mathrm{~mm} / \mathrm{d}$ ) from observations and from the 16-model ensemble of simulations for the present (1986-2005) and future (2080-2099) climates under the RCP4.5 and RCP8.5 scenarios; (b) the same results are presented here as in (a) but for the percentage changes for the two scenarios; (c) the same results are presented here as in (a) but for the precipitation ratio to total precipitation; and (d) the same results are presented here as in (c) but for the percentage changes for the precipitation amount.

the changes in the number of rainy days $(>0.1 \mathrm{~mm} / \mathrm{d})$ and the annual precipitation. The results from the RCP4.5 (Figure 3(a)) and RCP8.5 (Figure not shown) scenarios consistently indicate that the number of rainy days are projected to increase over the regions of northern China and the eastern part of Tibet Plateau but that the number of rainy days is projected to decrease over the regions of the western part of Tibet Plateau, the mid-lower reaches of Yangtze river, and the southeastern coast in China. However, large uncertainty is found over most parts of China. As opposed to the increase in annual precipitation, the area-averaged rainy days are reported to decrease by $0.3 \%$ in $\mathrm{RCP} 4.5$ and $1.7 \%$ in RCP8.5. An increase in the amount of precipitation but a decrease in the number of rainy days implies an increased intensity of rainfall events in China, which is supported by our calculation. The precipitation intensity is projected to increase by $9.3 \%$ in RCP4.5 and $16.7 \%$ in RCP8.5. These results suggest a high risk of flash floods and landslides in China under global warming scenario.

Because of the significant decrease in the number of days of trace rain events in China (Figure 3(b)), the trace rain amount exhibits a declining trend across the whole of China (Figure 2(b)), although its intensity is reported to increase (Figure not shown). In the RCP4.5 scenario, the area-averaged number of trace rain days is projected to decrease by $3.7 \%$, and its amount is projected to decrease by $3.3 \%$. A larger decrease can be observed in the RCP8.5 scenario, and $7.0 \%$ and $6.4 \%$ are reported for the number of trace rain days and its amount, respectively. This decrease in the number of trace rain days produces a negative contribution to the annual precipitation increase of the change in the trace rain amount (Figure 4(a)). There is an approximately $1.9 \%$ decrease in the area-averaged contribution in the RCP4.5 scenario, while an approximately $2.5 \%$ decrease occurs in the RCP8.5 scenario.

Compared with the change in trace rain, the regional feature is much more distinct for the change in light rain in China (Figure 2(c)). The MME results indicate that the light rain amount will significantly increase in northern China, especially in Northwest China, where a larger increase than other regions is expected, while it will significantly decrease in southern China. Considering China as a whole, the light rain amount exhibits an increase with respect to the present-day climate, with an increase by $1.8 \%$ in RCP4.5 and by $1.1 \%$ in RCP8.5. The changes in the light rain days are much similar to the changes in its amount (Figure 3(c)), with more days in northern China but fewer days in southern China. In addition, the intensity of light rain is projected to consistently increase across China (Figure not shown), which means that the change in the number of light rain days is the primary contributor to the change of its amount. Thus, the spatial distribution of the light rain contribution 

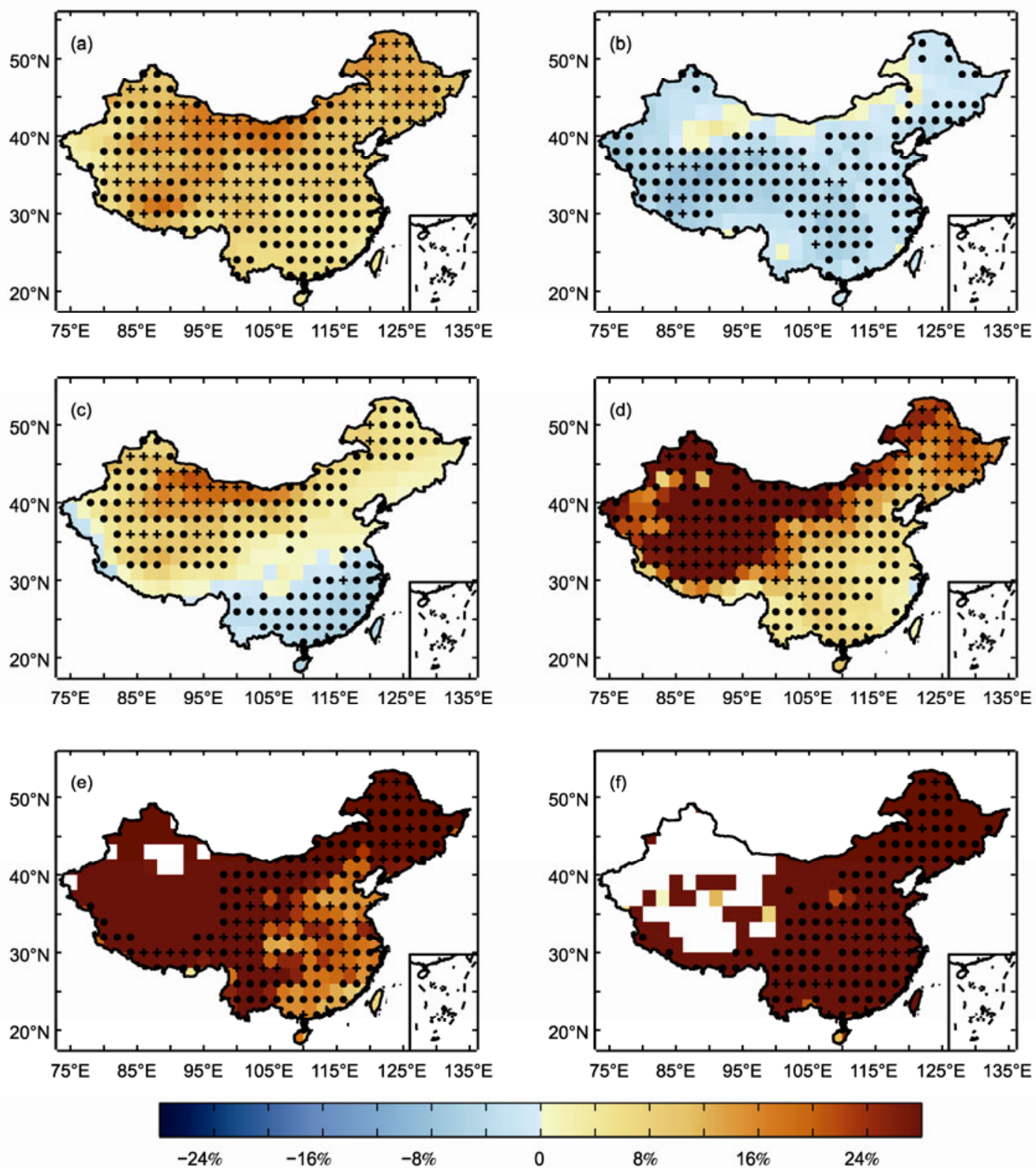

Figure 2 Projected precipitation changes from the period of 1986-2005 to the period of 2080-2099 from the 16-model ensemble in the RCP4.5 scenario. (a) Annual precipitation, (b) trace rain, (c) light rain, (d) medium rain, (e) large rain, and (f) heavy rain. $\bullet$ shows more than $67 \%$ (likely) of the models indicate a similar change as the MME, and + shows at least $90 \%$ (very likely) of the models indicate such a change. Blank regions denote no rainy events.

(Figure 4(b)) is very similar to the change in the number of days. The changes in the area-averaged light rain amount are contributed by $9.6 \%$ and $4.1 \%$ to the increase of the annual precipitation in RCP4.5 and RCP8.5, respectively.

By the end of the 21st century, the simulations indicate that the medium rain amount exhibits a significant increase across all of China (Figure 2(d)), with a larger increase in magnitude for northern China, especially in Northwest China. Both the changes in the number of medium rain days (Figure 3(d)) and the intensity of the medium rain events (Figure not shown) are reported to significantly increase, with a larger increase in northern China and a smaller increase in southern China. Due to increases in the number of days and the intensity of these rain events, the change in the medium rain amount exhibits a positive contribution to the increase of the annual precipitation across all of China. This change is quite obvious in the RCP8.5 scenario (Figure not shown). Compared with the present-day climate, the areaaveraged amount in the RCP4.5 and RCP8.5 scenarios increases by $12.0 \%$ and $16.7 \%$, respectively; the number of days increases by $11.3 \%$ and $15.3 \%$, respectively; the intensity increases by $3.4 \%$ and $6.5 \%$, respectively; and by $40.7 \%$ and $38.3 \%$ of the increased annual precipitation are reported due to the increase in the medium rain amount, respectively. These results are consistent across all the models as well as the MME. 

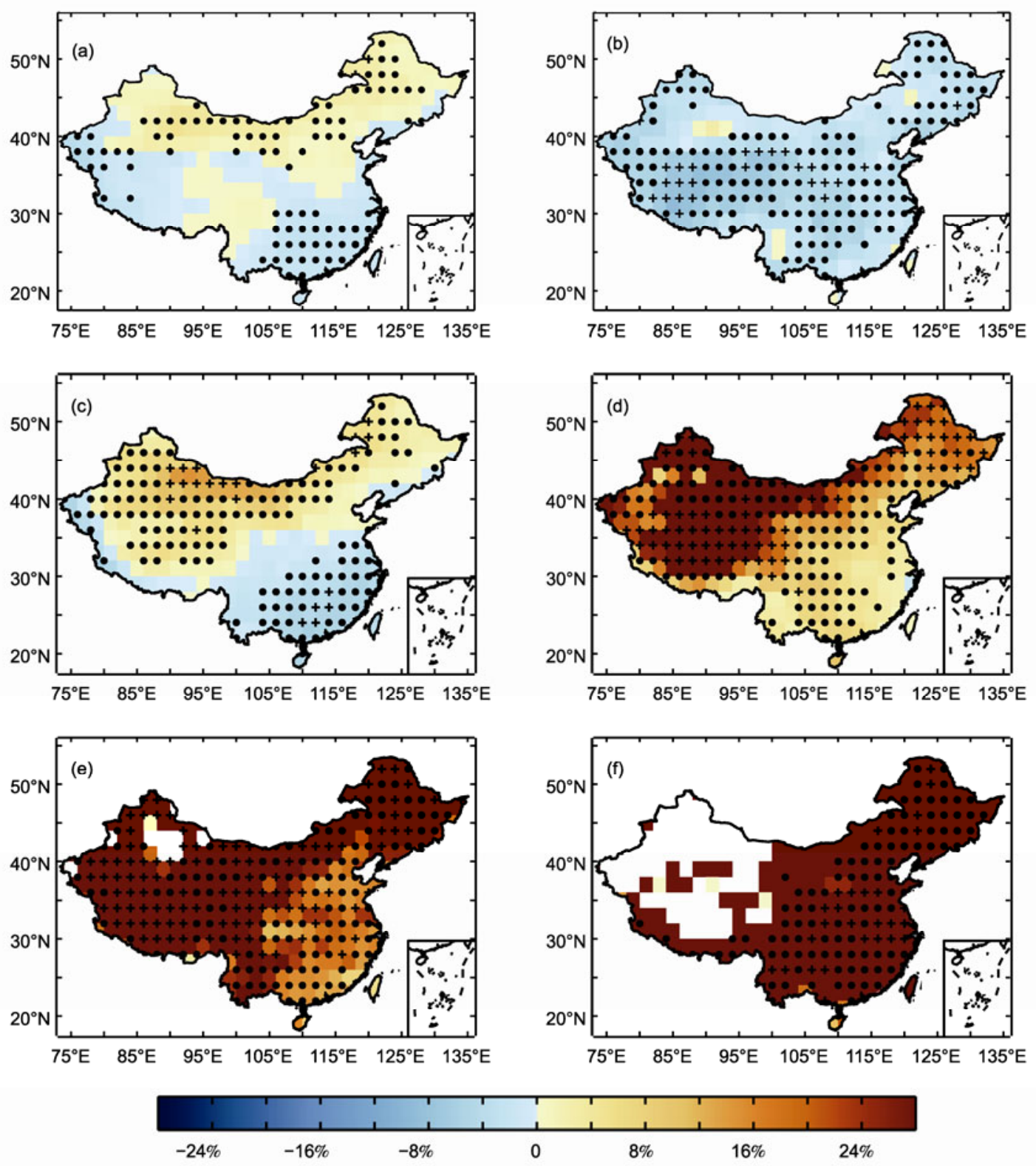

Figure 3 Same as in Figure 2, but for the rainy days (daily precipitation $>0.1 \mathrm{~mm} / \mathrm{d}$ ) and the number of days for five rainfall events.

Similar results are observed in the changes of the large rain. The large rain amount is projected to significantly increase (Figure 2(e)) because of the significant increases in its number of days (Figure 3(e)) and intensity (Figure not shown). The area-averaged number of days in China increases by $24.2 \%$, and the intensity increases by $10.0 \%$, resulting in a $25.3 \%$ increase in the large rain amount with respect to the present-day. Larger increases can be found in the RCP8.5 scenario, with a $41.7 \%$ increase in the large rain days, $18.3 \%$ increase in the intensity, and $44.4 \%$ increase in its amount. This significant increasing trend is obvious in all 16 CMIP5 models. Due to the increase in the large rain amount, the positive contribution is significant fraction of the increase of annual precipitation in China (Figure 4(d)).
In the RCP4.5 and RCP8.5 scenarios, the change in the area-averaged large rain amount will see increases of $31.6 \%$ and $35.0 \%$ to the increase of annual precipitation amount, respectively.

The change in heavy rain events is the most obvious. Projections from the RCP4.5 and RCP8.5 scenarios consistently indicate a significant increase in the heavy rain amount because of the significant increase in the number of days (Figure 3(f)) and the intensity (Figure not shown), which also produces a positive contribution to the increase of annual precipitation (Figure 4(e)). These changes are much greater in the RCP8.5 scenario, but they are smaller in the RCP4.5 scenario. The area-averaged days, intensity, and rain amount are projected to increase by $57.7 \%, 44.7 \%$, and 

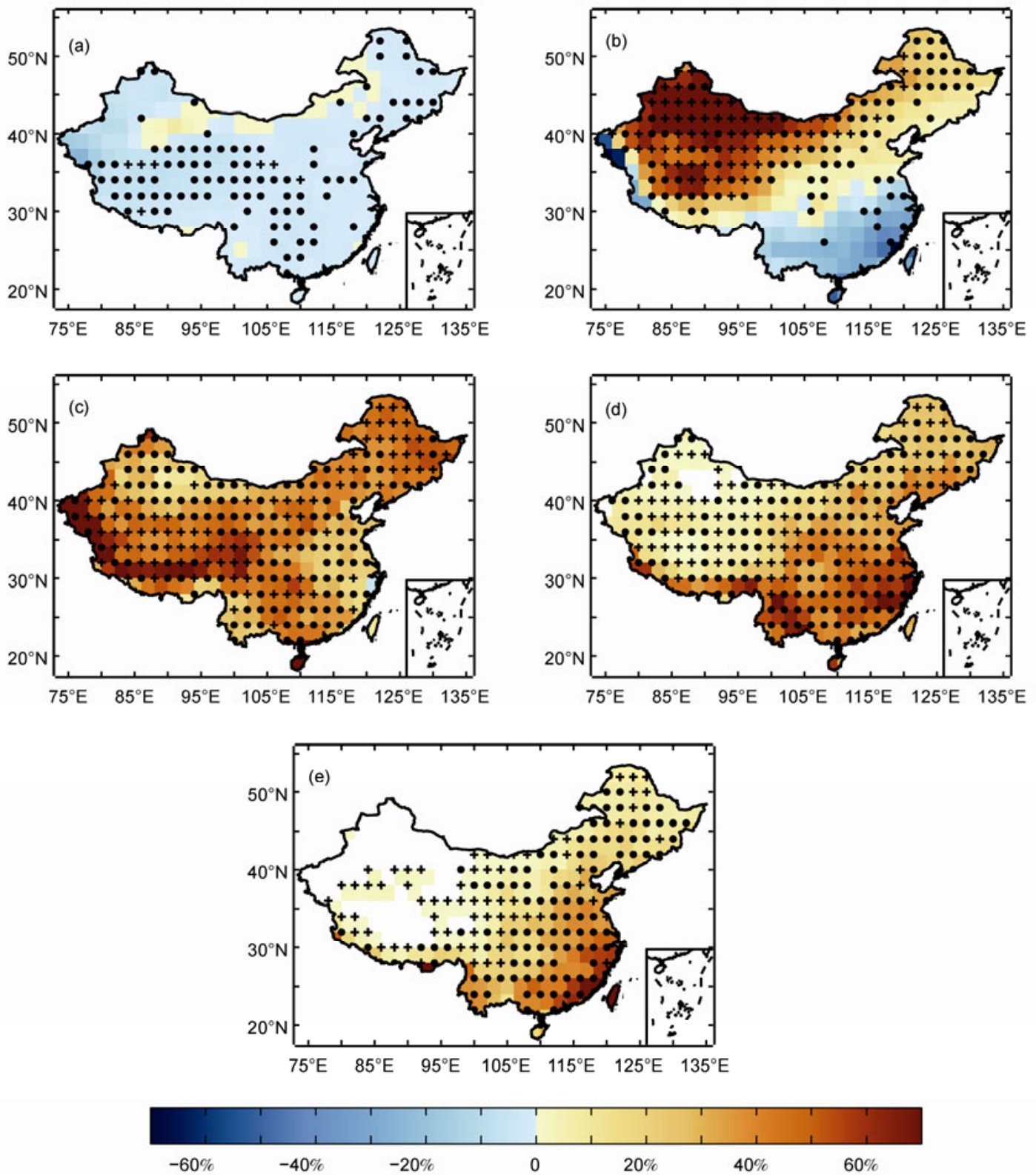

Figure 4 The spatial distribution of the projected contribution by the changes in the rainfall events to the increase of annual precipitation for the period 2080-2099 from the 16-model ensemble in the RCP4.5 scenario. (a) Trace rain, (b) light rain, (c) medium rain, (d) large rain, and (e) heavy rain. • shows more than $67 \%$ (likely) of the models indicate a similar change with the MME, and + shows at least $90 \%$ (very likely) of the models indicate such a change. Blank regions denote no rainy events.

$62.9 \%$, respectively, in the RCP4.5 scenario, and by $135.9 \%$, $98.8 \%$, and $153.7 \%$, respectively, in the RCP8.5 scenario. In addition, the increased rain amount contributes to the increase of annual precipitation by approximately $19.5 \%$ and $25.0 \%$ in RCP4.5 and RCP8.5, respectively. All the simulations indicate significant increasing trends that are similar to the MME results.

From the above analysis, it is clear that the precipitation and its related extremes are projected to significantly increase, but some differences are exhibited in the rainfall events of different-intensity categories under global warm- ing scenario. By the end of the 21 st century, all the simulations consistently project that the trace rain will significantly decrease across all of China. The increase of the annual precipitation in Northwest China is primarily due to increases in light rain. The increases in North and Northeast China are primarily due to increases in medium rain. In the region of southern China, the increases in the large rain and heavy rain play an important role in the increases in annual precipitation, while light rain events play a negative role. Compared with the projection in CMIP3, the CMIP5 simulations exhibit similar results, with a similar change magnitude 
in the days of intense precipitation, but with a much greater change in the magnitude of its intensity [15].

\section{Relationship between the changes in precipitation and temperature}

The relationship between the changes in precipitation and temperature has become an issue of concern, and researchers have started to pay significantly more attention to it in recent years. Both observations and simulations document that the global-mean precipitable water increases linearly with temperature at a rate of approximately $7 \% /{ }^{\circ} \mathrm{C}$ [31], which is much more obvious under future global warming scenarios. The simulations from 16 CMIP5 models indicate that, by the end of the 21 st century, the likely range of increase of surface air temperature is $1.5-7.5^{\circ} \mathrm{C}$ and that of annual precipitation is $2.2 \%-25.8 \%$ in China for the two scenarios considered. A least square fit to all the model projection yields a slope of $1.6 \% /{ }^{\circ} \mathrm{C}$, which is somewhat larger than the change in global-mean precipitation in the CMIP3 simulations [30]. In addition, a high correlation exists between the changes in the area-averaged precipitation and temperature in China, and the correlation is up to 0.47 , which is significantly above the $99 \%$ confidence level.

For trace rain, there is a consistent relationship between the changes in the number of days and temperature, with correlation coefficient of -0.51 , and the number of days is projected to decrease with a linear trend of $-1.3 \% /{ }^{\circ} \mathrm{C}$. The changes in light rain are much more complex. Its intensity is projected to increase $\left(0.3 \% /{ }^{\circ} \mathrm{C}\right)$ with the temperature increase, and their correlation coefficient can be up to 0.51 . However, there is no consistent relationship between the changes in the number of days and temperature, and their correlation coefficient is only -0.16 . For medium rain events, the MME exhibits increases with a $1.5 \% /{ }^{\circ} \mathrm{C}$ trend in the number of days and with a $0.9 \% /{ }^{\circ} \mathrm{C}$ trend with respect to its intensity, and the correlation coefficients with increased temperature among the models are reported to be 0.41 and 0.55 , respectively. For the intense precipitation, the relationships with temperature change are much more obvious. The correlation of temperature is up to 0.81 for the change in large rain days and is up to 0.83 for the change in heavy rain days. The rate of the increase in the number of days with surface warming in China is approximately $6.0 \% /{ }^{\circ} \mathrm{C}$ for large rain events and is approximately $27.3 \% /{ }^{\circ} \mathrm{C}$ for heavy rain events. We also calculate the relationships between the changes in intensity of intense precipitation and surface temperature. For large rain event, the intensity increases with temperature by $2.4 \% /{ }^{\circ} \mathrm{C}$ and their correlation is up to 0.74 . A greater increasing trend appears in the heavy rain intensity. Moreover, this trend increases by $19.7 \% /{ }^{\circ} \mathrm{C}$, and its correlation with temperature is up to 0.84. Additionally, further analysis indicates that the start day of intense precipitation is projected to be advanced and the end day of intense precipitation is projected to be delayed at the end of the 21 st century with respect to the present-day climate. Here, the start day is defined as the first day that the daily precipitation exceeds $25 \mathrm{~mm} / \mathrm{d}$ in one certain year and the end day is the last day that the intense precipitation event occurs. This means that the time interval between the first day and the last day that the intense precipitation event occurs is projected to be prolonged, with an increase by approximately 10 days in RCP4.5 and by approximately 17 days in RCP8.5 in China. The changes in the spatial extent that the intense precipitation event occurs in China are also estimated, and the results indicate that the spatial extent is projected to increase by approximately $5.0 \%$ and $8.7 \%$ in RCP4.5 and RCP8.5, respectively.

Positive relationships are indicated between the changes in precipitation and its related extremes with surface warming in China. However, a great deal of uncertainty exists between these relationships on the regional scale; therefore, more research is required.

\section{Future changes in atmospheric circulation over the East Asian region}

The seasonal variability in the number of days of precipitation and its related extremes are calculated from the observations in China in the target period 1986-2005 (Figure not shown), and the results indicate that the focused time period of precipitation and its related extremes mainly span from June to August. Thus, the following analysis will mainly focus on this summer season.

Figure 5(a) shows the spatial distributions of the changes in summer mean surface air temperature, sea level pressure, and $850-\mathrm{hPa}$ horizontal winds for the RCP4.5 scenario based on the 16-model ensemble over East Asia. Figure 5(b) shows the changes in the vertical integrated water vapor flux (integration from surface to $300 \mathrm{hPa}$ ) and the precipitable water content. From Figure 5, the summer surface air temperature increases over East Asia and the Pacific areas by the end of the 21 st century, but stronger surface warming is expected over East Asia. Accordingly, the sea level pressure over East Asia is projected to decrease, but it should rise over the Northwest Pacific. This increased land-sea contrast between East Asia and its adjacent ocean is much clearer in the RCP8.5 scenario (Figure not shown). Consequently, anomalous southerly winds prevail over East Asia. Meanwhile, northeasterly winds are projected to be stronger over this region in the high level of $100 \mathrm{hPa}$. In particular, for the region to the south of $30^{\circ} \mathrm{N}$, the increase in northerly winds is quite obvious (Figure not shown). This result implies that the East Asian summer monsoon will be much stronger at the end of the 21 st century with respect to the present-day climate. From the observations, two main water vapor sources for the precipitation processes in eastern China are the Indian Ocean and the tropical Western Pacific 

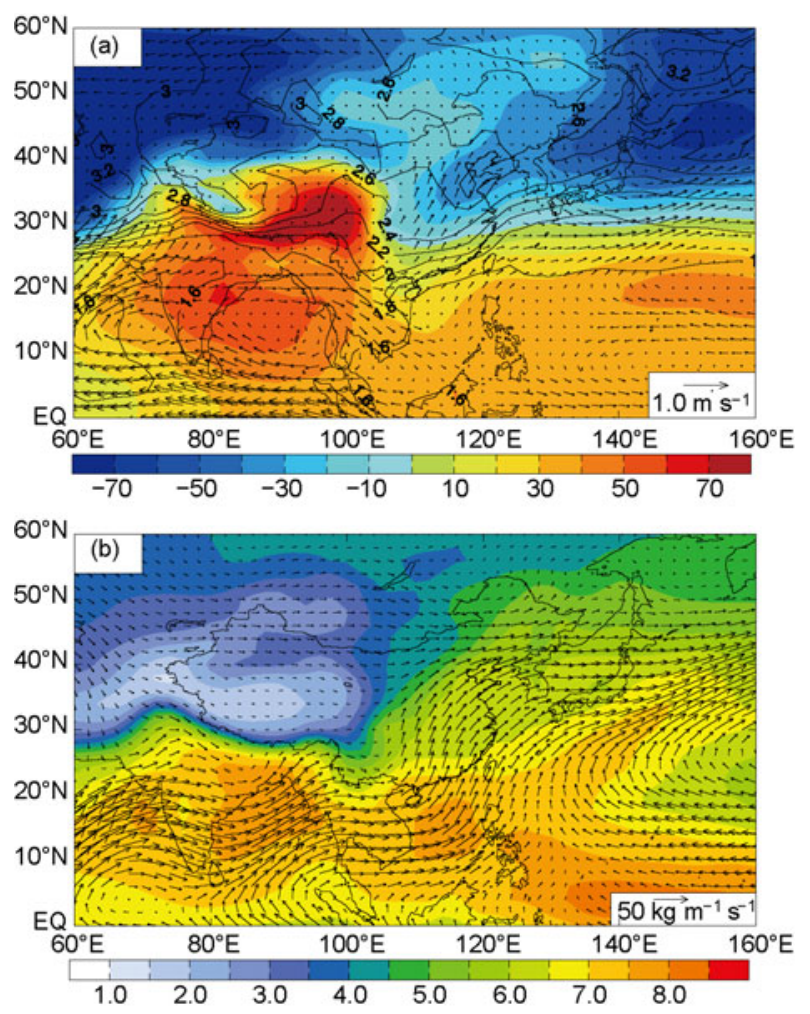

Figure 5 Projected changes in the summer large-scale circulations over East Asia in the period of 2080-2099 with respect to the period of 19862005 in the RCP4.5 scenario. (a) Changes in the sea level pressure (shading, Pa), 2-m air temperature (contour, ${ }^{\circ} \mathrm{C}$ ), and $850 \mathrm{hPa}$ horizontal winds (only the differences that are significant at a $95 \%$ confidence level are plotted); (b) changes in the precipitable water content (shading, $\mathrm{kg} / \mathrm{m}^{2}$ ) and the vertical integrated water vapor flux from the surface to $300 \mathrm{hPa}$.

[32]. These two sources become significantly stronger by the end of the 21st century because of the intensification of East Asian summer monsoon circulation that leads to a significant increase of the precipitable water content (Figure 5(b)). The MME results indicate a $16.6 \%$ increase in the area-averaged precipitable water content in RCP4.5 and a $36.4 \%$ increase in RCP8.5. Hence, the intensified East Asian summer monsoon could provide sufficient water vapor sources for the increase of precipitation and its related extremes over East China under global warming scenario.

Atmospheric stability is another important diver for the occurrences of precipitation and its related extremes. The $K$ index, a diagnostic factor for precipitation occurrence, has shown an ability to depict the stability of the atmosphere and has been widely used in the operational works. The large value of the $K$ index generally favors more occurrences of precipitation and its related extremes [15,33]. By the end of the 21st century, the projections from the MME results consistently indicate that the $K$ index over East Asia increases significantly in the two RCP scenarios, but with a greater increase in high-latitude than in low-latitude. This geographical distribution of change in the $K$ index is very similar with the change in the number of days of extreme rainfall events, with a larger increase in northern China and less of an increase in southern China. Considering China as a whole, all 16 CIMP5 models exhibit a consistent increase in the area-averaged $K$ index, and a $1.5^{\circ} \mathrm{C}$ increase is reported in the MME result of RCP4.5. A larger increase is exhibited in RCP8.5, and the MME indicates a $3.4^{\circ} \mathrm{C}$ increase. This significant increase in the $K$ index suggests that atmospheric stratification becomes much more unstable, thereby providing a beneficial background for the increases of precipitation and its extremes under global warming scenario.

\section{Conclusions}

The future potential changes in precipitation and its extremes are critical for the long-term social planning. The precipitation changes in China thus are investigated based on 16 simulations in the CMIP5 for the RCP4.5 and RCP8.5 scenarios, and the results consistently project that the rainfall events in China are reported to significantly increase and become much more extreme by the end of the 21 st century. Furthermore, the East Asian summer monsoon is projected to be much stronger under global warming scenario. The main conclusions are as follows:

(1) The analysis of the changes in precipitation indicates that, by the end of the 21st century, the annual precipitation is projected to significantly increase and the rainfall events will become much more extreme under global warming scenario. The number of days of trace rain is observed to significantly decrease across all of China, while a significant increase is expected in the number of days of medium rain, large rain, and heavy rain. The number of light rain days is projected to increase in northern China but decrease in southern China. Unlike the changes in the rainfall days, the intensity is projected to significantly increase for all events by the 16 models. Further analysis indicates that the contributions to the increase of annual precipitation by the rainfall events of different-intensity categories exhibit similar distributions with the changes in the number of rainfall days, with a negative contribution for trace rain in China, and a positive contribution for medium rain, large rain, and heavy rain, and a positive contribution in northern China for light rain but a negative contribution in southern China.

(2) The analysis on the relationships between the changes in precipitation and temperature exhibits a significantly negative correlation in the days of trace rain and significantly positive correlations in the days of medium rain, large rain, and heavy rain, and no significant correlation in the days of light rain. By the end of the 21st century, the days of trace rain are reported to decrease with a linear trend of $-1.3 \% /{ }^{\circ} \mathrm{C}$ when the two scenarios are considered. However, the days of medium rain, large rain, and heavy rain are projected to significantly increase with temperature at a rate of $1.5 \% /{ }^{\circ} \mathrm{C}, 6.0 \% /{ }^{\circ} \mathrm{C}$, and $27.3 \% /{ }^{\circ} \mathrm{C}$, respectively. Accordingly, the annual precipitation increases significantly 
with temperature, with the regressed linear trend of $1.6 \% /{ }^{\circ} \mathrm{C}$.

(3) The analysis of the changes in the large-scale circulations indicates that the East Asian summer monsoon is projected to be much stronger. This increase in intensity leads to significantly higher water vapor content in East China that is sourced from the Indian Ocean and the tropical Pacific Ocean. Meanwhile, atmospheric stratification becomes much more unstable. These changes in large-scale circulations provide a favorable background for the occurrence of precipitation under global warming scenario.

With scientific advancement, the models involved in CMIP5 are all substantially improved in many aspects, e.g. resolution, experimental design, and emission scenarios [26]. Despite the good performance in simulating the precipitation climatology in China, most models still underestimate the annual precipitation and its variability, which will be discussed in a subsequent paper. From the projections of this study, we can see that the models indicate consistent changes in the annual precipitation and its related extremes, but a large uncertainty still exists because of the differences in the parameter schemes, response to the external forcing, and other reasons, for the different models. All models project increases in the annual precipitation and its related extremes, but large differences are projected in the various magnitudes of these increases. Currently, research involving climate prediction and projection using high-resolution RCMs is underway [34-36]. Compared with the couple models, RCMs exhibit improved performance in simulating the regional climate features. However, some discrepancies can be observed in climate projection between the RCMs and the couple models. For example, the annual precipitation and its related extremes are projected to significantly increase across all of China at the end of the 21 st century by the couple models; however, the results from RegCM3 indicate that the precipitation and its related extremes decrease over the regions of Southwest China and the Tibetan Plateau, but they increase over the other regions [18]. Hence, more research based on high-resolution RCMs is quite necessary in future climate projection analyses that can be used to further identify the uncertainty between the RCMs and the couple models.

The author would like to thank three anonymous reviewers for suggestions that improved this paper. This work was supported by the National Basic Research Program of China (2012CB955401), the "Strategic Priority Research Program-Climate Change: Carbon Budget and Relevant Issues" of the Chinese Academy of Sciences (XDA05090306), the National Natural Science Foundation of China (41275075) and the CAS-CSIRO Cooperative Research Program (GJHZ1223).

1 Meehl G A, Stocker T F, Collins W D, et al. Climate change 2007: The physical science basis. In: Solomon S, Qin D, Manning M, et al, eds. Contribution of Working Group 1 to the Fourth Assessment Report of the Intergovernmental Panel on Climate Change. Cambridge, United Kingdom and New York: Cambridge University Press, 2007. $1-18$
2 Chen H P, Sun J Q. How the "best" models project the future precipitation change in China. Adv Atmos Sci, 2009, 26: 773-782

3 Jiang D B, Zhang Y, Sun J Q. Ensemble projection of $1-3^{\circ} \mathrm{C}$ warming in China. Chin Sci Bull, 2009, 54: 3326-3334

4 Zhang Y, Sun J Q. Model projection of precipitation minus evaporation over China. Acta Meteorol Sin, 2012, 26: 376-388

5 Lu R Y, Fu Y H. Intensification of East Asian summer rainfall interannual variability in the twenty-first century simulated by 12 CMIP3 coupled models. J Clim, 2010, 23: 3316-3331

6 Sun Y, Ding Y H. A projection of future changes in summer precipitation and monsoon in East Asia. Sci China Earth Sci, 2010, 53: 284300

7 Wang $\mathrm{H} \mathrm{J}$, Zeng Q C, Zhang X $\mathrm{H}$. The numerical simulation of the climatic change caused by $\mathrm{CO}_{2}$ doubling. Sci China Ser B, 1993, 36: 451-462

8 Zhou B T. Simulation and projection of Hadley circulation in coupled climate models (in Chinese). Clim Environ Res, 2012, 17: 339-352

9 Chen H P, Sun J Q, Chen X L. Future changes of drought and flood events in China under global warming scenario. Atmos Oceanic Sci Lett, 2012, 6: 8-13

10 Jiang D B, Fu Y H. Climate change over China with a $2^{\circ} \mathrm{C}$ global warming (in Chinese). Chin J Atmos Sci, 2012, 36: 234-246

11 Buhe C. Simulation of the future change of East Asian monsoon climate using the IPCC SRES A2 and B2 scenarios. Chin Sci Bull, 2003, 48: 1024-1030

12 Luo Y, Zhao Z C, Xu Y, et al. Projections of climate change over China for the 21st century. Acta Meteorol Sin, 2005, 19: 401-406

13 Chen H P, Sun J Q, Chen X L. The projection and uncertainty analysis of summer precipitation in China and the variations of associated atmospheric circulation (in Chinese). Clim Environ Res, 2012, 17: $171-183$

14 Jiang Z H, Chen W L, Song J, et al. Projection and evaluation of the precipitation extremes indices over China based on seven IPCC AR4 coupled climate models (in Chinese). Chin J Atmos Sci, 2009, 33: 109-120

15 Chen H P, Sun J Q, Chen X L, et al. CGCM projections of heavy rainfall events in China. Int J Climatol, 2012, 32: 441-450

16 Sun J Q, Wang H J, Yuan W, et al. Spatial-temporal features of intense snowfall events in China and their possible change. J Geophys Res, 2010, 115: D16110

17 Xu Y L, Zhang Y, Lin E D, et al. Analyses on the climate change responses over China under SRES B2 scenario using PRECIS. Chin Sci Bull, 2006, 51: 2260-2267

18 Shi Y, Gao X J, Giorgi F, et al. High resolution simulation of changes in different-intensity precipitation events over China under global warming (in Chinese). Adv Clim Change Res, 2010, 6: 164-169

19 Shi Y, Gao X J, Wang Y G, et al. Simulation and projection of monsoon rainfall and rain patterns over eastern China under global warming by RegCM3. Atmos Oceanic Sci Lett, 2009, 2: 308-313

20 Gao X J, Shi Y, Giorgi F. A high resolution simulation of climate change over China. Sci China Earth Sci, 2011, 54: 462-472

21 Gao X J, Shi Y, Zhang D F, et al. Climate change in China in the 21st century as simulated by a high resolution regional climate model. Chin Sci Bull, 2012, 57: 1188-1195

22 Ding Y H, Ren G Y, Zhao Z C, et al. Detection, causes and projection of climate change over China: An overview of recent progress. Adv Atmos Sci, 2007, 24: 954-971

23 Wang H J, Sun J Q, Chen H P, et al. Extreme climate in China: Facts, simulation and projection. Meteorol Zeitschrift, 2012, fast track doi: 10.1127/0941-2948/2012/0330

$24 \mathrm{Xu} \mathrm{C} \mathrm{H,} \mathrm{Xu} \mathrm{Y.} \mathrm{The} \mathrm{projection} \mathrm{of} \mathrm{temperature} \mathrm{and} \mathrm{precipitation} \mathrm{over}$ China under RCP scenarios using a CMIP5 multi-model ensemble. Atmos Oceanic Sci Lett, 2012, 5: 527-533

$25 \mathrm{Xu} \mathrm{Y,} \mathrm{Xu} \mathrm{C} \mathrm{H.} \mathrm{Preliminary} \mathrm{assessment} \mathrm{of} \mathrm{simulations} \mathrm{of} \mathrm{climate}$ change over China by CMIP5 multi-models. Atmos Oceanic Sci Lett, 2012, 5: 489-494

26 Taylor K E, Stouffer B J, Meehl G A. An overview of CMIP5 and the experiment design. Bull Amer Meteorol Soc, 2012, 93: 485-498

27 Moss R H, Edmonds J A, Hibbard K A, et al. The next generation of 
scenarios for climate change research and assessment. Nature, 2010, 463: 747-756

28 Jiang D B, Wang H J, Lang X M. Evaluation of East Asian climatology as simulated by seven coupled models. Adv Atmos Sci, 2005, 22: $479-495$

29 Xu C H, Shen X Y, Xu Y. An analysis of climate change in East Asia by using the IPCC AR4 simulation (in Chinese). Adv Clim Change Res, 2007, 3: 287-291

30 Sun Y, Solomon S, Dai A, et al. How often will it rain? J Clim, 2007, 20: 4081-4098

31 Trenberth K E, Dai A, Rasmussen R M, et al. The changing character of precipitation. Bull Amer Meteorol Soc, 2003, 84: 1205-1217

32 Wang H J, Chen H P. Climate control for southeastern China mois- ture and precipitation: Indian or East Asian monsoon? J Geophys Res, 2012, 117: D12109

33 Chen H P, Sun J Q, Fan K. Decadal features of heavy rainfall events in eastern China. Acta Meteorol Sin, 2012, 26: 289-303

34 Gao X J, Shi Y, Zhang D F, et al. Uncertainties of monsoon precipitation projection over China: Results from two high resolution RCM simulations. Clim Res, 2012, 52: 213-226

35 Wang H J, Yu E T, Yang S. An exceptionally heavy snowfall in Northeast China: Large-scale circulation anomalies and hindcast of the NCAR WRF model. Meteorol Atmos Phys, 2011, 113: 11-25

36 Yu E T, Wang H J, Sun J Q. A quick report on a dynamical downscaling simulation over China using the nested model. Atmos Oceanic Sci Lett, 2010, 3: 325-329

Open Access This article is distributed under the terms of the Creative Commons Attribution License which permits any use, distribution, and reproduction in any medium, provided the original author(s) and source are credited. 